\title{
Experimental Evidence for Vortex-Glass Superconductivity in Y-Ba-Cu-O
}

\author{
R. H. Koch, V. Foglietti, W. J. Gallagher, G. Koren, A. Gupta, and M. P. A. Fisher \\ IBM Research Divison, T. J. Watson Research Center, P.O. Box 218, \\ Yorktown Heights, New York 10598 \\ (Received 7 June 1989)
}

\begin{abstract}
We demonstrate experimentally the existence of a continuous phase transition between a normal and a true superconducting phase (with zero linear resistivity) in epitaxial films of $\mathrm{Y}-\mathrm{Ba}-\mathrm{Cu}-\mathrm{O}$ in strong magnetic fields, $H \gg H_{c 1}$. The nonlinear $I-V$ curves show scaling behavior near the transition and the relevant critical exponents are extracted. These exponents are consistent with values expected for freezing into a superconducting vortex-glass phase.
\end{abstract}

PACS numbers: $74.60 . \mathrm{Ge}, 74.70 . \mathrm{Vy}$

Since the discovery of high-temperature superconductivity in 1986, the nature of the mixed state in strong magnetic fields $H>H_{c 1}$ and low temperatures has been an unresolved question. Much of the recent $I-V^{1}$ and magnetic-susceptibility ${ }^{2}$ data have been interpreted in terms of the Anderson-Kim flux-creep model, ${ }^{3}$ or extensions thereof ${ }^{4,5}$ which include effects of pinning but ignore important collective effects of the vortex lines. ${ }^{6}$ In these models, the linear resistance $R_{L} \equiv \lim _{I \rightarrow 0} V / I$ is predicted to drop rapidly (exponentially) upon cooling but always remain nonzero (except possibly at $T=0$ ): A true superconducting mixed-state phase, with $R_{L} \equiv 0$, is not present. On the other hand, ${ }^{7}$ data from torsionaloscillator experiments have been constructed as evidence for the freezing of a vortex-fluid (i.e., normal) phase into an ordered Abrikosov-flux lattice. Without bulk pinning, though, the Abrikosov lattice will have a nonzero resistivity due to flux flow. In the presence of pinning, however, Larkin and Ovchinnikov ${ }^{8}$ have shown that the long-range crystalline order of the Abrikosov lattice is destroyed, precluding freezing into a truly ordered solid phase.

Recently, by taking into account both pinning and collective effects of the vortex lines, one of us (M.P.A.F.) has argued that at low $T$ in a bulk system the vortex lines should freeze into a new "vortex-glass" phase. ${ }^{9-11}$ In sharp contrast to the flux-creep model, the vortexglass phase is predicted to be a true superconductor with $R_{L} \equiv 0$. An equilibrium phase boundary at a well defined temperature in the $H-T$ plane is expected, separating the normal phase at high $T$ from the superconducting glass phase.

The purpose of this Letter is to demonstrate experimentally that in $\mathrm{Y}-\mathrm{Ba}-\mathrm{Cu}-\mathrm{O}$ samples in a strong magnetic field there in fact does exist strong evidence of a transition at a well defined temperature from a resistive state into a superconducting state $\left(R_{L}=0\right)$ upon cooling. Analysis of nonlinear $I-V$ curves enables us, in addition, to extract scaling behavior and related critical exponents near the transition. Although these measurements do not probe details of the low-temperature phase (beyond the fact that $R_{L}=0$ ), the exponents are consistent with values expected theoretically for freezing into the vortex-glass phase.

A continuous (or second-order) superconductor-tonormal phase transition leaves a distinctive signature in the nonlinear $I-V$ characteristics. A general formulation of the scaling at and near such a transition has recently been carried out. ${ }^{12}$ The basic idea is that physical quantities near the transition can be expressed as the appropriate powers of a diverging coherence length $\xi$ and coherence time $\xi^{z}$. The coherence length diverges with an exponent $v, \xi \sim\left|T-T_{g}\right|^{-v}$, at the transition and $z$ is the dynamical exponent. Assuming isotropic scaling (which is expected for the vortex-glass transition) $9,11,13$, the dc $I-V$ characteristics for a $d$-dimensional sample should then scale as $\left(\phi_{0}=h / 2 e\right)$

$$
E(J) \approx J \xi^{d-2-z} \tilde{E}_{ \pm}\left(J \xi^{d-1} \phi_{0} / k_{B} T\right),
$$

where $J$ is the current density and $E$ is the electric field. Note that in the scaling functions $\tilde{E}_{ \pm}(x), J$ is scaled by a characteristic current density

$$
J_{0}=k_{B} T / \phi_{0} \xi^{d-1},
$$

which vanishes as $T \rightarrow T_{g}$. For $T<T_{g}, J_{0}^{-} \equiv J_{0}$ is essentially the "critical-current" density of the superconducting phase. The scaling function in the normal phase, $\tilde{E}_{+}(x)$, goes to a constant as $x \equiv J / J_{0} \rightarrow 0$, implying a linear resistivity which vanishes as

$$
\rho_{L} \sim\left(T-T_{g}\right)^{v(z+2-d)} .
$$

This is a generalization of the Aslamazov-Larkin meanfield result for the $\boldsymbol{H}=\mathbf{0}$ superconductor-normal transition, $^{14}$

$$
\rho_{L} \sim\left(T-T_{c}\right)^{(4-d) / 2},
$$

wherein $z=2$ and $v=\frac{1}{2}$.

Equation (1) dictates that for $x \rightarrow \infty, \tilde{E}_{ \pm}(x)$ $\sim x^{(z+2-d) /(d-1)}$, which gives a power-law $I-V$ curve for $T=T_{g}$

$$
E\left(J ; T=T_{g}\right) \approx J^{(z+1) /(d-1)} .
$$


The result for the Kosterlitz-Thouless transition, appropriate to thin-film superconductors ${ }^{15}$ with $H=0$, $E \sim J^{3}$, is a special case of Eq. (3) with $z=d=2$. The scaling function in the superconducting phase, $\tilde{E}_{-}(x)$, vanishes (exponentially in $1 / x$, in 3D) as $x \rightarrow 0$ (so that $\rho_{L}=0$ ). Thus, in sum, we expect the $I-V$ curve for $T$ near $T_{g}$ to be a power law as in (3) for large enough currents, crossing over to Ohmic behavior at low currents for $T>T_{g}$, and crossing over to exponentially vanishing dissipation for $T<T_{g}$. For $T<T_{g}$, this implies a negative curvature on a $\log I-\log V$ plot, in sharp contrast to the flux-creep prediction, $V \sim \sinh \left(I / I_{0}\right)$, which always has positive curvature.

The above scaling forms are general and can be used to analyze the data presented below, regardless of the details of the superconducting phase. If, as we believe, the low- $T$ phase is a $3 \mathrm{D}$ vortex-glass phase, then ${ }^{9}$

$$
V_{-}(x) \sim \exp \left(-1 / x^{\mu}\right)
$$

for $x \rightarrow 0$ (with a glass exponent $0 \leq \mu \leq 1$ ) and (rough) constraints can be put on the critical exponents $v$ and $z$. Specifically, as in magnetic spin glasses, meanfield theory, which is valid for $d \geq 6$, gives $v=\frac{1}{2}$ and $z=4$. For $d=3$ we expect $z>4$, as in the Ising spin glass, and a first order in $\epsilon=6-d$ expansion gives ${ }^{13}$ $v=1 / 2+5 \epsilon / 24$, which suggests $v \sim 1-2$ in 3D.

There are (at least) two important length scales in this problem: (i) the average distance $l$ between the vortex lines $l \simeq\left(\phi_{0} / \pi H\right)^{1 / 2}$ and (ii) the correlation length $\xi$ which can be estimated from $J_{0}$ using (2). The critical regime for the vortex-glass to normal transition requires, at the very least, $\xi \gtrsim l$, and is thus more accessible in large fields. In the experiments described below, which are on films with thickness $t=0.4 \mu \mathrm{m}$, the 3D critical regime requires, moreover, $\xi \lesssim t$ (which is satisfied in the regime measured below). Since the vortex-glass phase is, strictly speaking, not expected to exist in $2 \mathrm{D}, 9,12$ the crossover to $2 \mathrm{D}$ behavior for $\xi \gtrsim t$ is probably quite complicated.

We have made measurements on epitaxial thin films of $\mathrm{YBa}_{2} \mathrm{Cu}_{3} \mathrm{O}_{y}$ deposited using laser ablation ${ }^{16}$ onto $\mathrm{SrTiO}_{3}$ (001) substrates. The resistivity of the samples at $100 \mathrm{~K}$ and zero field was typically $1 \times 10^{-6} \Omega \mathrm{m}$. Ion milling was used to define true four-terminal contact patterns with center stripes that were nominally $0.4 \times 8 \times 40 \mu \mathrm{m}^{3}$ in size. The small sample size was chosen to minimize error from any possible spatial inhomogeneities in the film. The films were predominantly $c$ axis up and had critical-current densities of more than $10^{10} \mathrm{~A} / \mathrm{m}^{2}$ at 77 $\mathrm{K}$ in zero applied field. The magnetic field was applied in the direction of the $c$ axis using a superconducting magnet.

Identification of a phase transition depends critically on the shape of the $I-V$ curve at constant temperature, as discussed above. Joule heating increases the local temperature of the sample and its immediate surroundings compared to the sample-mounting block (which was temperature regulated). The primary source of Joule heating was from the $\sim 1-\Omega$ current contacts to the sample. This effect, if uncorrected, can substantially change the shape of the $I-V$ curve since we are measuring a very temperature-dependent part of the curve. To circumvent this potential error at larger currents, we recorded the $I-V$ curves using a bidirectional current sweep at $11 \mathrm{~Hz}$.
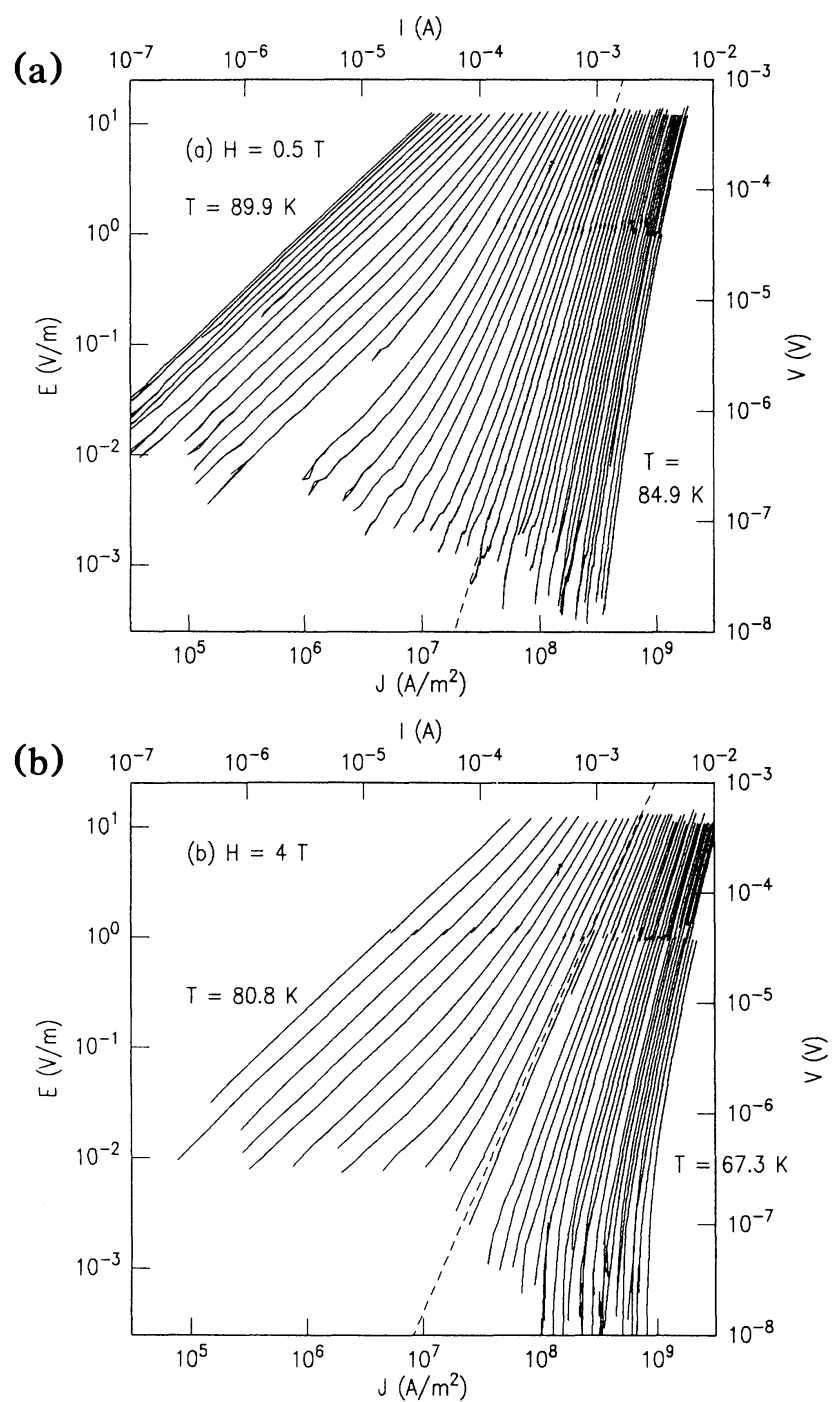

FIG. 1. $I-V$ curves at constant $T$ for $H=$ (a) $0.5 \mathrm{~T}$ and (b) $4 \mathrm{~T}$. The curves differ by temperature intervals of 0.1 and 0.3 $\mathrm{K}$, respectively. At each temperature, a high- and a lowcurrent-sweep $I-V$ curve was measured and spliced together at $40 \mu \mathrm{V}$. Any change in the average temperature of the sample will result in a disconnection between the upper and lower $I-V$ curves. In the upper right-hand corner of (b), crowding of the $I-V$ curves from Joule heating can be seen. The high-current $I-V$ curve taken at a temperature regulator setting of $67.3 \mathrm{~K}$ matches the low-current $I-V$ curve taken at a setting of $66.7 \mathrm{~K}$. The shape of each isotherm, however, is unaffected. 
TABLE I. Measured exponents at three field values.

\begin{tabular}{ccccc}
\hline $\begin{array}{c}\text { Applied } \\
\text { field } \\
H(\mathrm{~T})\end{array}$ & $\begin{array}{c}z \text { measured } \\
\text { from } I-V \\
\text { curve }\end{array}$ & $\begin{array}{c}v \text { measured } \\
\text { from } J_{0}^{+}(T)\end{array}$ & $\begin{array}{c}v(z+2-d) \\
\text { from } \\
\text { measured } z, v\end{array}$ & $\begin{array}{c}v(z+2-d) \\
\text { measured } \\
\text { from } \rho_{L}(T)\end{array}$ \\
\hline 2 & 4.9 & 1.7 & 6.6 & 6.2 \\
3 & 5.0 & 1.6 & 6.4 & 6.5 \\
4 & 4.7 & 1.8 & 6.4 & 6.5 \\
\hline \hline
\end{tabular}

Since the wave-form repeat time was much shorter than the measured thermal time constant of more than $10 \mathrm{sec}$, heating only changed the average temperature of the sample, but not the shape of the $I-V$ curve. The temperature rise was measured to be less than $0.1 \mathrm{~K}$ for biascurrent sweeps of $I_{\mathrm{pp}}=4 \mathrm{~mA}$, but $0.6 \mathrm{~K}$ for $I_{\mathrm{pp}}=20 \mathrm{~mA}$. Most of the data were taken with $I_{\mathrm{pp}}<4 \mathrm{~mA}$. At low $T$ varying the frequency of measurement from 100 to 0.1 $\mathrm{Hz}$ had an essentially unobservable effect on the $I-V$ curves. Below $0.03 \mathrm{~Hz}$, the shape of the curve was distorted by heating.

Figure 1 plots a series of $I-V$ curves at constant temperature for $H=0.5$ and $4 \mathrm{~T}$. Not shown are similar data for $H=1,2$, and $3 \mathrm{~T}$. The field was applied with the sample in the normal state and the data were taken as the temperature was reduced. For each field, at a single well defined temperature $T$ (dashed line in Fig. 1), the $I-V$ curves show a power-law behavior at all but the highest currents measured. For higher $T$, the $I-V$ curves have positive curvature, and at low $I$, a crossover to an Ohmic regime with $R_{L} \neq 0$. For lower $T$, the $I-V$ curves show negative curvature with a voltage which plummets rapidly upon reducing $I$, extrapolating to $R_{L}=0$. In sum, the experimental data have precisely the form expected for a vortex-glass transition, provided we identify the temperature at which the power-law behavior occurs (dashed line) as the transition temperature $T_{g}(H)$. In the following, we define this temperature as $T_{g}$.

We emphasize that the $I-V$ curves for $T<T_{g}$ are inconsistent with the flux-creep model, $V \sim \sinh \left(I / I_{0}\right)$, which predicts a positive curvature on a $\log I-\log V$ plot. ${ }^{17}$

The dynamical critical exponent can be extracted directly from the $I-V$ data. At $T_{g}$, we find $\partial \log E /$ $\partial \log J=2.9 \pm 0.2$, which upon using (3) gives $z=4.8$ \pm 0.2 in good agreement with our expectations for a vortex-glass transition in 3D. The values of $H, T_{g}$, and $z$, and other measured parameters are tabulated in Table I. The phase boundary in the $H-T$ plane, separating the normal and superconducting phases, is shown in Fig. 2. We note that $T_{g}$ is not appreciably different from the superconducting "transition" temperature measured in the usual way, i.e., when $V$ is less than some experimentally observable value.

The crossover current density, $J_{0}$, can also be extracted directly from the experimental $I-V$ curves. Above $T_{g}$, for $J<J_{0}^{+}$, the $I-V$ curve is characterized by a linear resistance $R_{L}(T)$. At larger current densities, the curve

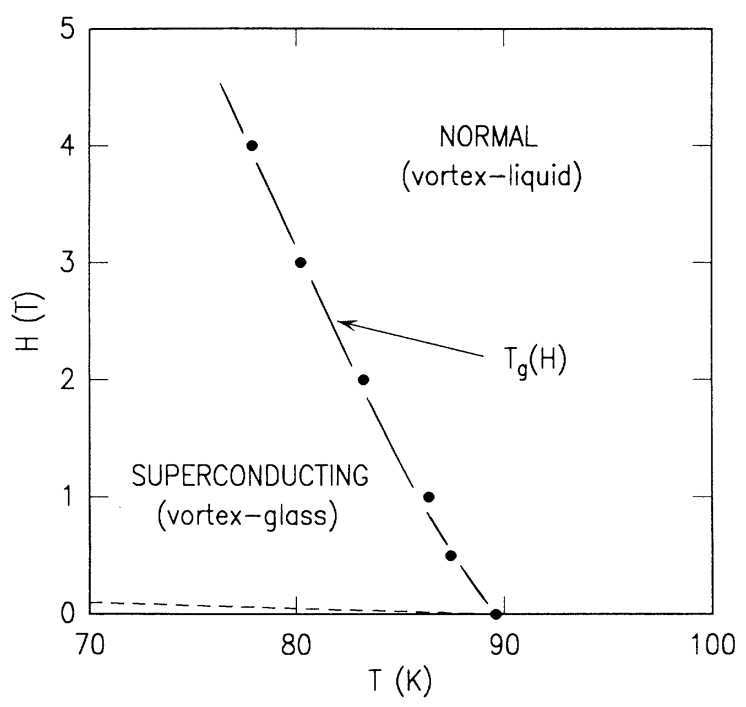

FIG. 2. The equilibrium phase boundary between the normal and superconducting phase in the $H-T$ plane. The dashed line represents the expected Meissner phase.

is seen to be asymptotic to a power law parallel to the $I$ $V$ curve at $T_{g}$. We can quantify this crossover by defining $J_{0}^{+}$as the current at which $\partial \log E / \partial \log J=10 / 9$ (other choices for this constant between 1 and 3 would only change the magnitude of $J_{0}^{+}$, i.e., not the scaling behavior). Figure 3 plots $J_{0}^{+}(T)$ vs $\left(T-T_{g}\right) / T_{g}$. Here $T_{g}$ is not a fitting parameter, but the value measured independently above. For $H>1 \mathrm{~T}$, we find reasonable power-law behavior. Using (2), in this regime, gives an exponent $v=1.7$. An unknown systematic error in our measurement of $T_{g}$ of $0.2 \mathrm{~K}$ would change this estimate of $v$ by $25 \%$.

For $T \leq T_{g}$ the $I-V$ curve is seen to crossover from the

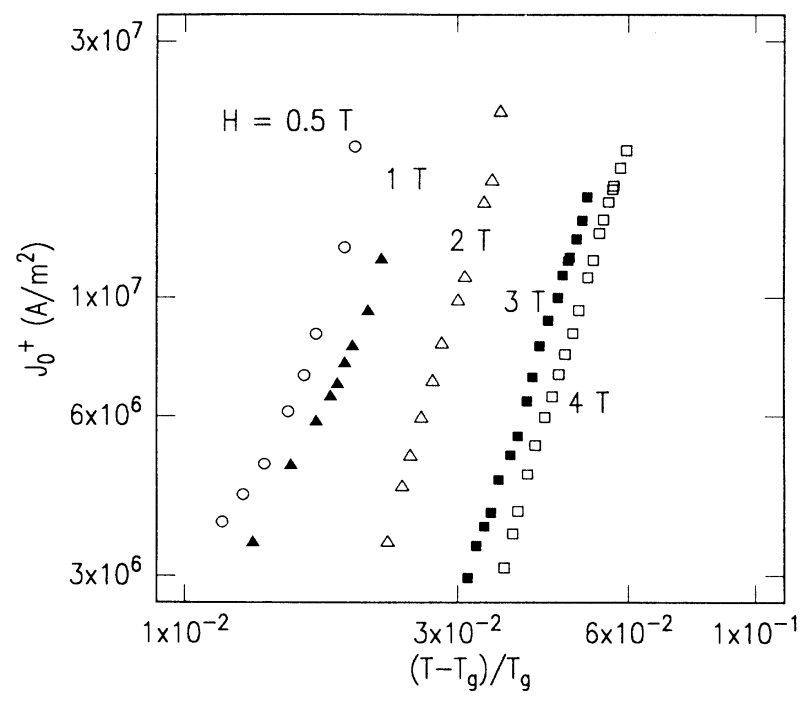

FIG. 3. $J_{0}^{+}(T)$ vs $\left(T-T_{g}\right) / T_{g}$ for five values of $H$. 


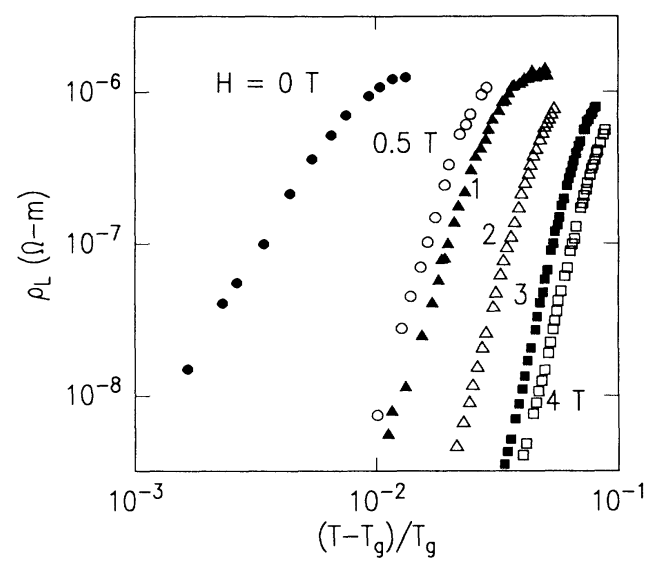

FIG. 4. $\rho_{L}(T)$ vs $\left(T-T_{g}\right) / T_{g}$ for six values of $H$.

critical power law at currents larger than $J_{0}^{-}$, to an exponential behavior at lower $J$, consistent again with expectations from the scaling theory. We define $J_{0}^{-}$as that value of $J$ where $\partial \log E / \partial \log J=5$. Extracting $v$ from $J_{0}^{-} \sim\left(T_{g}-T\right)^{2 v}$ we find a high-field value of roughly 1.5-2.0 in agreement with that obtained from the temperature scaling of $J_{0}^{+}$.

For $H=1$ and $0.5 \mathrm{~T}$, the curves in Fig. 3 show significant curvature, perhaps due to errors in estimating $T_{g}$. Alternatively, this may be because the true critical regime has not been reached yet. At low fields one expects a narrow critical regime due to the large vortexline separation $l$. Indeed, roughly estimating $\xi$ from (2) using a midrange value of the measured $J_{0}^{+}(T)$ gives $\xi$ in the range from 40 to $160 \mathrm{~nm}$. To be in the critical regime requires $\xi>l$, which is well satisfied for high fields, $H=4 \mathrm{~T}$, where $l=20 \mathrm{~nm}$, but not at low fields, $H=0.5$ $\mathrm{T}$, where $l=60 \mathrm{~nm}$. Exponents obtained at large $H$ are thus more reliable.

A consistency check of the scaling behavior near the phase transition is to analyze the temperature dependence of the linear resistivity $\rho_{L}$ for $T>T_{g}$, deduced from the $I-V$ curves. Figure 4 plots $\rho_{L}(T)$ vs $\left(T-T_{g}\right) /$ $T_{g}$ and the exponents are listed in Table I. Scaling implies $\rho_{L} \sim\left(T-T_{g}\right)^{v(z+2-d)}$. Using the high-field value of $v=1.7$, we find a value of $z=4.8$, in excellent agreement with the estimate of $z$ obtained independently from the $I-V$ curve at $T_{g}$.

Below $T_{g}$, the vortex-glass model predicts an $I-V$ curve of the form of Eq. (4). The curves in Fig. 1 show negative curvature for $T<T_{g}$ as predicted by this equation, but it is difficult to extract a numerical estimate of the exponent $\mu$ from our data. Fitting the shape of the $I-V$ curve by (4) allows us to estimate that $\mu \approx 0.4 \pm 0.2$.

In summary, our data demonstrate the existence of a second-order phase transition between a normal and a true superconducting $\left(R_{L} \equiv 0\right)$ state at a well defined temperature $T_{g}$ in epitaxial films of $\mathrm{Y}-\mathrm{Ba}-\mathrm{Cu}-\mathrm{O}$ in large fields. We expect a similar transition in single crystals and other high-temperature superconductors. The measured exponents of the transition are consistent with those expected for a transition into a vortex-glass superconductor.

We thank R. B. Laibowitz, B. Oh, and A. M. Torressen for experimental assistance. We also thank D. S. Fisher, P. M. Horn, S. Von Molnar, and T. K. Worthington for many productive conversations. We are particularly indebted to D. A. Huse for his invaluable help in formulating the scaling theory sketched herein. This work was partially supported by the U.S. Office of Naval Research Contract No. N00014-88-C-0439.

${ }^{1}$ T. T. M. Palstra, B. Batlogg, L. F. Schneemeyer, and J. V. Waszczak, Phys. Rev. Lett. 61, 1662 (1988).

${ }^{2}$ A. P. Malozemoff, T. K. Worthington, Y. Yeshurun, and F. Holtzberg, Phys. Rev. B 38, 7203 (1988).

${ }^{3}$ P. W. Anderson and Y. B. Kim, Rev. Mod. Phys. 36, 39 (1964).

${ }^{4}$ Y. Yeshurun and A. P. Malozemoff, Phys. Rev. Lett. 60, 2202 (1988).

${ }^{5}$ M. Tinkham, Phys. Rev. Lett. 61, 1658 (1988).

${ }^{6}$ In the flux-creep approach a vortex line (or a bundle of lines) is modeled as a single (zero-dimensional or point) "particle" moving in a random potential with energy scale $U$. In a bulk 3D superconductor though, vortex lines are of course one-dimensional objects, comprised of many adjoining "segments" which feel different pinning environments, yet cannot move independently. In M. P. A. Fisher, Phys. Rev. Lett. 62, 1415 (1989), account is taken of the collective behavior of these many segments and the collective behavior of the many vortex lines, leading to a prediction of the vortex-glass phase.

${ }^{7}$ P. L. Gammel, L. F. Schneemeyer, J. V. Waszczak, and D. J. Bishop, Phys. Rev. Lett. 61, 1666 (1988).

${ }^{8}$ A. I. Larkin and Yu. N. Ovchinnikov, J. Low Temp. Phys. 34, 409 (1979).

${ }^{9}$ Fisher, Ref. 6.

${ }^{10}$ C. Ebner and D. Stroud, Phys. Rev. B 31, 165 (1985).

${ }^{11}$ S. John and T. Lubensky, Phys. Rev. B 34, 4815 (1986).

${ }^{12}$ D. A. Huse, D. S. Fisher, and M. P. A. Fisher (unpublished).

${ }^{13}$ A. Houghton and M. A. Moore, Phys. Rev. B 38, 5045 (1988).

${ }^{14}$ L. G. Aslamazov and A. I. Larkin, Phys. Lett. 26A, 238 (1968).

${ }^{15}$ A. F. Hebard and A. T. Fiory, Phys. Rev. Lett. 50, 1603 (1983).

${ }^{16}$ G. Koren, A. Gupta, E. A. Giess, A. Segmuller, and R. B. Laibowitz, Appl. Phys. Lett. 54, 1054 (1989).

${ }^{17}$ The data also do not fit cleanly into a flux-creep plus fluxflow scenario. The low-T I-V data, which have negative curvature, could, in this scenario, only be due to a crossover from flux creep to flux flow. This appears inconsistent with the fact that at higher $T$, yet in the same current-density range $\left(\sim 10^{8}\right.$ $\mathrm{A} / \mathrm{m}^{2}$ ), the data show Ohmic behavior due to flux creep. 